\title{
Guided Imagery Intervention does not Affect Surgical Outcome of Patients Undergoing laparoscopic Cholecystectomy: A Multi-Centre, Randomised Controlled Study
}

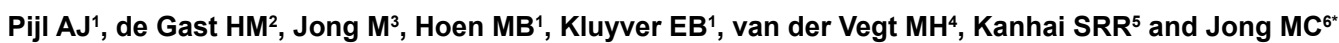

${ }^{1}$ Department of Anesthesiology, Slotervaartziekenhuis, Amsterdam, The Netherlands ${ }^{2}$ Department of Anesthesiology, Rode Kruis Ziekenhuis, Beverwijk, The Netherlands

${ }^{3}$ Department of Health Sciences, Mid Sweden University, Sundsvall, Sweden

${ }^{4}$ Department of Anesthesiology, Zaans Medisch Centrum, Zaandam, The Netherlands

${ }^{5}$ Department of Anesthesiology, Sint Lucas Andreas Ziekenhuis, The Netherlands

${ }^{6}$ Department of Nutrition and Health, Louis Bolk Institute, Driebergen, The Netherlands

\begin{abstract}
Objective:To investigate if a "non-pharmacological" intervention with guided imagery could reduce postoperative analgesic consumption, pain perception and preoperative anxiety, compared to standard care, in patients undergoing laparoscopic cholecystectomy (LC).
\end{abstract}

Methods: A randomized controlled study with two parallel groups was performed at two hospital departments of anesthesiology. A total of 140 patients ( $\geq 18$ years) that were scheduled for LC were randomized to either receive guided imagery $(\mathrm{N}=70)$ or standard care instructions $(\mathrm{N}=70)$ as a control group. Patients in the guided imagery group were provided a CD to practice guided imagery once a day, 7 days prior to surgery. Primary outcome measurement was post-operative analgesic consumption. Secondary outcomes were preoperative anxiety, post-operative self-rated pain, patient satisfaction and adverse events.

Results: Of 140 patients that were randomized, 95 patients completed the study, 43 in the guided imagery group and 52 in the control group. Both groups were comparable at baseline with respect to demographic data. Compliance with intervention was fairly good as $77 \%$ of the patients had listened to the CD according to instructions. No significant differences $(p=0.34)$ were observed for postoperative morphine use between the intervention $(15.8 \pm 18.5 \mathrm{mg})$ and control group $(12.5 \pm 13.6 \mathrm{mg})$. Secondary outcomes such as preoperative anxiety (APAIS: $15.2 \pm 5.9 \mathrm{vs.} 16.4 \pm 5.9$; $p=0.36)$ ), postoperative pain (VAS: $3.4 \pm 1.8$ vs. $3.0 \pm 1.8 ; p=0.31$ ) and patient satisfaction (PSQ: $4.1 \pm 0.9$ vs. $3.9 \pm$ $0.8 ; p=0.47$ ) also demonstrated no significant differences. No adverse events were reported in both groups.

Conclusion: A short preoperative guided imagery intervention demonstrated no additional beneficial effects compared to standard care for patients undergoing LC. It therefore seems not to be as simple as to provide patients with a CD before surgery in order to effectively self-manage postoperative pain.

Keywords: Surgery; Post-operative pain; Preoperative anxiety; Non-pharmacological intervention; Cholecystectomy; Mind-body intervention; Randomized controlled trial

\section{Introduction}

Effective control of pain after surgery is of importance as severe postoperative pain has been shown to impair wound healing, prolong hospital stays and lead to the onset of chronic pain syndromes [1-3]. Anxiety before surgery is the most common predictor for postoperative pain [4]. Good surgical pain management may therefore benefit from strategies that minimize pre-operative anxiety and stress. Various pharmaceutical regimens to reduce pre-operative anxiety have been devised. However, variable patient response and side effects often limit the efficacy of these approaches [5]. Recent studies demonstrate that non-pharmacological interventions, such as mind-body interventions and distraction therapies, may emerge as promising intervention strategies to reduce anxiety, stress and pain. It has been demonstrated that hypnotherapy sessions significantly alleviate preoperative anxiety, reduce postoperative pain intensity and improve wound outcome [6,7]. In addition, virtual reality techniques may be useful in reducing pain and anxiety in burn injury patients [8]. Previous studies have shown that relaxation with guided imagery may reduce anxiety in surgical patients undergoing cholecystectomy and in paediatric surgical patients, although further rigorous studies are warranted in this area
$[9,10]$. In guided imagery, the imagination is used to relax and to distract attention away from pain and anxiety. For example, patients may imagine themselves on a beach or on a mountainside to stimulate relaxation and distract attention from pain; or they may imagine the pain itself and manipulate that image to alter the pain sensation [11]. The aim of the present study was to examine the possible beneficial effects of guided imagery in overall surgical pain management. Therefore, a large multi-center randomized controlled clinical study was designed and patients undergoing laparoscopic cholecystectomy (LC) were selected to investigate the effects of guided imagery on surgical outcome.

*Corresponding author: YJong MC, Department of Nutrition and Health, Louis Bolk Institute, Driebergen, The Netherland, Tel: +31 343 523860; Fax +31 343515 611; E-mail: m.jong@louisbolk.nl

Received: August 18, 2016; Accepted: September 01, 2016; Published: September 07, 2016

Citation: Pijl AJ, de Gast HM, Jong M, Hoen MB, Kluyver EB, et al. (2016) Guided Imagery Intervention does not Affect Surgical Outcome of Patients Undergoing laparoscopic Cholecystectomy: A Multi-Centre, Randomised Controlled Study. J Pat Care 2: 119. doi: 10.4172/2573-4598.1000119

Copyright: (c) 2016 Pijl AJ, et al. This is an open-access article distributed unde the terms of the Creative Commons Attribution License, which permits unrestricted use, distribution, and reproduction in any medium, provided the original author and source are credited. 
Citation: Pijl AJ, de Gast HM, Jong M, Hoen MB, Kluyver EB, et al. (2016) Guided Imagery Intervention does not Affect Surgical Outcome of Patients Undergoing laparoscopic Cholecystectomy: A Multi-Centre, Randomised Controlled Study. J Pat Care 2: 119. doi: 10.4172/25734598.1000119

\section{Methods}

\section{Study design}

A randomized, single-blinded, multi-centre pilot study with two parallel groups was conducted in The Netherlands (NL). The clinical study was approved by the Institutional Review Board of the Slotervaart Hospital, Amsterdam, NL. If patients agreed to take part in the study, they gave written informed consent. The study was conducted in accordance with the regulation in NL, the Declaration of Helsinki and in adherence to the ICH guideline for Good Clinical Practice. The reporting of this randomised clinical trial is according to the updated guidelines of the Consolidated Standards of Reporting Trials [12]. The anesthesiologists, surgeons and nursing staff that were responsible for handling of the patients in the study, were all blinded to group allocation. Patients were asked not to reveal their group allocation.

\section{Study population}

It was decided to study surgery outcomes after LC as this surgery is commonly performed and an earlier study showed promising results [9]. Eligible patients were all adults aged $\geq 18$ years scheduled for LC with a good understanding of the Dutch language. Exclusion criteria were patients with psychiatric disorders and patients who had no CD player or were not willing to perform the guided imagery exercises. Half of the eligible patients $(n=70)$ was recruited at the Slotervaart Hospital (Amsterdam, NL) and the other half $(\mathrm{n}=70)$ at the Red Cross Hospital (Beverwijk, NL). The first patient was included in July 2009 and the last patient completed the study in September 2011.

\section{Study and operation procedure}

Eligible patients were informed about the study and invited to participate by an anaesthesiologist at the outpatient clinics. After written consent, patients were randomly allocated to the intervention (guided imagery) group $(n=70)$ or a control group $(n=70)$. All patients in the study underwent a standard elective LC under general anaesthesia. The patients received temazepam $10 \mathrm{mg}$, paracetamol $1000 \mathrm{mg}$ and diclofenac $100 \mathrm{mg}$ in the premedication, unless contraindicated. Anaesthesia was induced with propofol $1-2 \mathrm{mg} / \mathrm{kg}$, sufentanil $0.5 \mathrm{ug} /$ $\mathrm{kg}$ and rocuronium $0.6 \mathrm{mg} / \mathrm{kg}$. The anaesthesia was maintained with sufentanil and sevoflurane in air $\left(\mathrm{FiO}_{2}\right.$ of $\left.40 \%\right)$ with atleast 1.2 endtidal concentration of sevoflurane.

In the recovery room after surgery, patients received i.v. morphine 1-3 mg until analgesia was adequate, i.e., Visual Analogue Scale (VAS) $\leq 4$. Thereafter the patients received a Patient Controlled Intravenous Analgesia (PCIA) pump with morphine. The bolus dose was $1 \mathrm{mg}$ of morphine and the lock-out interval was $6 \mathrm{~min}$. The maximum dose per $4 \mathrm{~h}$ was $40 \mathrm{mg}$ of morphine. Furthermore the patients were prescribed Paracetamol $1000 \mathrm{mg} 4$ times daily, and diclofenac $50 \mathrm{mg} 3$ times daily.

\section{Intervention}

For the purpose of this study, a $\mathrm{CD}$ on guided imagery was developed by the Van Praag Institute (Utrecht, the Netherlands) in close collaboration with the clinical experts and patients. Prior to the start of the study, the developed guided imagery $C D$ was validated among a group of 40 patients undergoing surgery at the Slotervaart Hospital (Amsterdam, the Netherlands) with respect to the use, content and possible beneficial effects of the guided imagery and relaxation exercises on the CD.

Patients allocated to the intervention group were provided a guided imagery $\mathrm{CD}$ in an envelope, together with the standard information of the hospitals, to take home. They were asked to perform the exercise on the $\mathrm{CD}$ once a day during a period of 7 days before surgery. The exercise was about $20 \mathrm{~min}$ in length each day. At day 7 and day 3 prior to surgery, patients were contacted by telephone to remind them to listen to the $\mathrm{CD}$. The control group was not provided with a $\mathrm{CD}$, but received an envelope with standard information only on how to prepare for surgery.

\section{Outcome parameters}

The primary outcome was to determine whether a nonpharmacological intervention, i.e., relaxation with guided imagery, added to the standard perioperative analgesic and anxiolytic pharmacotherapy program, could reduce post-operative analgesic consumption, PCIA (mg morphine in $24 \mathrm{~h}$, as read from the display of the Gemstar pump).

Secondary outcome parameters were changes in preoperative anxiety (Amsterdam Preoperative Anxiety and Information Scale, APAIS; day of the operation), postoperative self-rated pain (VASscore/NRS-numeric rating scale; at least 4 times in the first $24 \mathrm{~h}$ ), patient(s) satisfaction (The Patient Satisfaction Questionnaire, PSQ 18 the day after surgery) and numbers of reported adverse events $[13,14]$.

\section{Sample size}

The study was powered based on the assumption that the postoperative use of morphine could be reduced with one-third in the guided imagery group compared to the control group. With a onesided level of significance of 0.05 and a power of $80 \%$, the number of patients in each group was calculated as 50 . Hence, to control for dropout, 70 patients were recruited for each group, 140 patients in total.

\section{Randomisation}

Patients were stratified per centre, therefore two separate randomisation list were generated using a Random Allocation Software Program with a random block size of 10 in order to guarantee a balanced allocation. The Guided imagery intervention and control (standard care) group were assigned to intervention $\mathrm{A}$ or intervention $\mathrm{B}$ by the study coordinator. Each included patient received an individual study number ranging from 0 to 150 . The study numbers were sequentially allocated to the patients in the order of inclusion in the study per centre. The randomisation list was kept confidential by the study coordinator until all data were completely entered into the database.

\section{Statistical methods}

All study data were recorded into case report forms (CRFs) on a continuous basis by the anaesthesiologists at the hospitals. The completed original CRFs were collected by the study coordinator and forwarded to the Louis Bolk Institute for data entry into the study data base and to the Mid Sweden University for statistical analysis. The analysis was based on the full analysis data set (FAS), including all patients that were randomized, operated according to the study operation procedure, performed the exercise at least once (intervention group) and for which at least one of the primary or secondary variables was documented.

Missing values for the VAS/NSR scores were replaced by the mean values of the participants non-missing values. Regular descriptive statistics were calculated with regard to demographical and categorical data and mean scores and standard deviations of the continuous variables using SPSS (version 19.0). Student's t-test or Chi-square test, were used to compare mean scores of the outcome variables in the guided imagery intervention and control group. 
Citation: Pijl AJ, de Gast HM, Jong M, Hoen MB, Kluyver EB, et al. (2016) Guided Imagery Intervention does not Affect Surgical Outcome of Patients Undergoing laparoscopic Cholecystectomy: A Multi-Centre, Randomised Controlled Study. J Pat Care 2: 119. doi: 10.4172/25734598.1000119

Page 3 of 5

\section{Results}

A flow diagram of patients included and excluded in the study is shown in Figure 1. A total of 140 patients were randomised, 70 in each group. In the control group, a total of 18 patients (18/70, $25.7 \%)$ dropped out. Of these 18 drop outs, nine patients $(9 / 18)$ underwent acute scheduled operation, in four patients $(4 / 18)$ the operation was cancelled, two patients $(2 / 18)$ were operated otherwise, one $(1 / 18)$ patient received other postoperative pain medication than morphine, one patient $(1 / 18)$ was operated in another hospital and one patient dropped out because study instructions were lost. In the guided imagery group, a total of 27 patients $(27 / 70=38.6 \%)$ dropped out. For eight patients $(8 / 27)$ this was due to an acute scheduled operation, in five patients $(5 / 27)$ the operation did not take place, five patients $(5 / 27)$ indicated that they did not listen to the CD because either the $\mathrm{CD}$ player or $\mathrm{CD}$ was broken, four patients did not listen to the $\mathrm{CD}$ $(4 / 27)$, two patient $(2 / 27)$ received other postoperative medication, two patients $(2 / 27)$ were operated otherwise and one patient $(1 / 27)$ withdrew without a reason. For the full set analysis, 96 patients were included (Guided Imagery: 43, Control: 52).

As shown in Table 1, the majority of patients included in the study were female. Both groups were comparable at baseline with respect to demographic data, since no significant differences were observed between the guided imagery and control group with respect to gender, comorbidity, age and body mass index.

Compliance with the intervention was fairly good. Ten patients $(10 / 43,23 \%)$ had listened to the CD up to three times. The majority $(28 / 43,65 \%)$ listened from four up to seven times to the CD and $12 \%$ (5/43) eight times or more. The primary efficacy parameter was the difference in postoperative analgesics use, through the PCIA. As shown in Table 2, no (statistical) differences were observed with respect to morphine use between the two groups.

Concerning the secondary efficacy parameter preoperative anxiety, no significant difference were observed in the total APAIS sum scores between the guided imagery and control group (Table 2). Similar results were found for the specific anxiety domain score within the APAIS (guided imagery: $9.4 \pm 4.3$ versus control: $10.2 \pm 4.3$, $\mathrm{p}=0.41$ ). Other secondary parameters such as the postoperative VAS/NSR scores (day of surgery and day after surgery) for pain and patient satisfaction also demonstrated no significant differences between the two groups. No adverse events were reported by patients in both groups.

Additional subgroup analysis with respect to different age categories demonstrated no significant differences between the guided
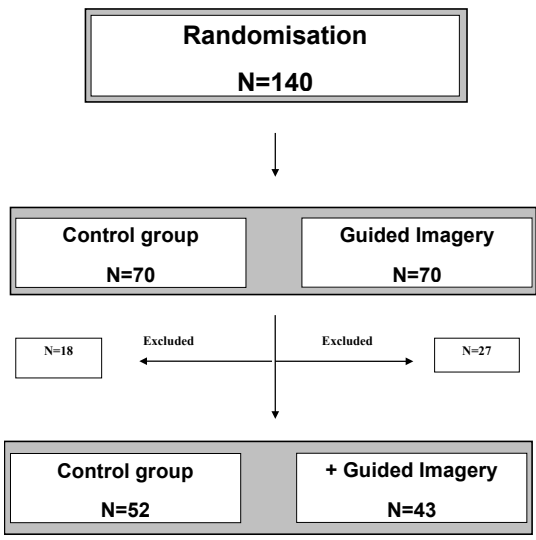

Figure 1: Flow diagram of patients.

\begin{tabular}{|c|c|c|c|}
\hline Characteristics & $\begin{array}{c}\text { Guided Imagery } \\
\mathbf{n = 4 3}\end{array}$ & $\begin{array}{c}\text { Control } \\
\mathbf{n = 5 2}\end{array}$ & p-value \\
\hline Female (\%) & 77 & 75 & 0.52 \\
\hline Male (\%) & 23 & 25 & 0.52 \\
\hline Comorbidity (\%) & 44 & 38 & 0.22 \\
Age (years) & $52 \pm 14.8$ & $495 \pm 14.3$ & 0.43 \\
\hline BMI & $28.2 \pm 5.1$ & $28.0 \pm 4.3$ & 0.88 \\
\hline
\end{tabular}

Full-set analyses values are either expressed as $\%$ of total or as mean $\pm S D$. $P$-value of the Chi-square or student t-test indicated no significant differences between the guided imagery and control group

Table 1: Demographics.

\begin{tabular}{|c|c|c|c|}
\hline Parameters & Guided Imagery $\boldsymbol{n = 4 3}$ & Control $\boldsymbol{n = 5 2}$ & $\boldsymbol{p}$-value \\
\hline Morphine (mg) & $15.8 \pm 18.5$ & $12.5 \pm 13.6$ & 0.34 \\
\hline APAIS (total sum) & $15.2 \pm 5.9$ & $16.4 \pm 5.9$ & 0.36 \\
\hline $\begin{array}{c}\text { VAS score (day of surgery) } \\
\text { VAS score (day 1) }\end{array}$ & $3.4 \pm 1.8$ & $3.0 \pm 1.8$ & 0.31 \\
\hline PSQ (general satisfaction) & $2.5 \pm 1.6$ & $2.47 \pm 1.7$ & 0.82 \\
\hline
\end{tabular}

Full-set analyses values are either expressed as $\%$ of total or as mean \pm SD P-value of the Students t-test indicated no significant differences between the guided imagery and control group on all outcome parameters

\section{Table 2: Outcome parameters.}

imagery and control group with respect to the primary outcome parameter $(p=0.211)$. Furthermore, women reported significantly more anxiety prior to surgery than men $(\mathrm{p}=0.014)$ and they were also less satisfied with treatment (general satisfaction) than men $(p=0.007)$. In the control group, a higher degree of anxiety (APAIS sum score) was significantly correlated with a higher degree of pain on the day of surgery (pearson correlation $\mathrm{r}=0.47, \mathrm{p}=0.001$ ) and the day after surgery $(\mathrm{r}=0.49, \mathrm{p}<0.001)$. Anxiety was not found to be a predictor of postoperative pain in the intervention group (day of surgery $r=0.18$, $\mathrm{p}=0.246$; day after surgery $\mathrm{r}=0.27, \mathrm{p}=0.097$ ).

\section{Discussion}

The present study failed to demonstrate an overall beneficial effect of guided imagery on postsurgical outcomes of patients undergoing LC. No significant differences were observed in postoperative analgesics, pain and satisfaction of LC patients after a preoperative guided imagery intervention compared to LC patients with standard care only. In line with these findings, the 7 day guided imagery intervention was not found to significantly reduce preoperative anxiety. These findings are in contrast to the beneficial effects of "non-pharmacological" interventions as reported for LC patients in other studies $[9,15,16]$. A study by Holden-Lund observed stress-relieving effects of guided imagery in patients undergoing cholecystectomy but was performed in a very small number of patients $(\mathrm{N}=24)$. Another small scale pilot study $(\mathrm{N}=13)$ by Fernandez et al. [16] observed a reduction of anxiety and postoperative analgesics in LC patients that received an acceptancebased psychological intervention. A recent more rigorous and larger randomised study in LC patients $(\mathrm{N}=75)$ by Broadbent et al. [15] demonstrated a significant decrease in perceived stress and improved wound healing with guided imagery compared to standard care. In the study, the guided imagery intervention included an individual meeting with a health psychologist who informed each patient on the negative impact of stress on surgical outcome and how relaxation exercises such as guided imagery could help reduce stress.

Patients were individually instructed in deep breathing techniques and guided imagery exercises and also performed home exercises using 
Citation: Pijl AJ, de Gast HM, Jong M, Hoen MB, Kluyver EB, et al. (2016) Guided Imagery Intervention does not Affect Surgical Outcome of Patients Undergoing laparoscopic Cholecystectomy: A Multi-Centre, Randomised Controlled Study. J Pat Care 2: 119. doi: 10.4172/25734598.1000119

a CD. As part of the intervention, the CD also contained an exercise for the patients to perform in the week after surgery to support recovery [15]. Patients in the control group were not informed about how stress and relaxation may affect surgical outcome. The differences in outcome between the Broadbent study and the present study could thus be explained by the different ways the guided imagery interventions were designed. In the present study, patients were simply instructed to listen to the exercise on the $\mathrm{CD}$ in the week before surgery. No additional individual meetings took place to inform patients about the possible benefits of relaxation on surgical outcome. The objective was primarily to investigate whether a simple and feasible guided imagery intervention could positively affect surgical outcomes, as routinely there are no possibilities in public hospitals for patients to have individual sessions with psychologists to prepare for surgery.

The additional instructions in the Broadbent study may have created expectancy about the efficacy of guided imagery in patients receiving the intervention, which was not present in the control group. Such an "expectancy" bias was not likely to occur in the present study and could explain the lack of effect in the present study. The lack of effect as observed in the present study could also be explained by the fact that the specific type of $\mathrm{CD}$ with guided imagery exercises as used in the present study was not appropriate for this target group. However, the CD as used in this study was specifically developed for this purpose together with all involved stakeholders (clinicians, nurses, patients) prior to the start of the study. The CD was pretested in 40 patients before its use in the study. It was evaluated as appropriate for its purpose and almost all patients recommended to provide this $\mathrm{CD}$ to patients before undergoing surgery. It therefore seems unlikely that another $\mathrm{CD}$ with other guided imagery exercises would have yield significant different effects between the two study groups.

The current study has it strengths and limitations. Strengths were that the study was single blinded, multi-centre, pragmatic in design and with highly comparable study groups. It is therefore expected that the findings can be generalized to LCs in other public hospital settings as well. One of the major limitations was the high dropout rate, 39\% in the guided imagery and $26 \%$ in the control group. The higher dropout in the guided imagery group was solely explained by the nine randomised patients that for several reasons had not listened to the CD. Another determinant contributing to the high dropout rate was that especially during the summer period, patients already scheduled for operation were called in acutely (within 1-3 days) due to cuts in the waiting list. These patients were not able to follow the instruction of the study, as time preceding surgery was too short.

Furthermore, surgery dates were delayed or cancelled and patients were rescheduled to other hospitals. All these internal and external factors have to be taken into account when performing such a pragmatic study. Due to the high dropout rate, the number of 50 patients as planned to complete the study in each group was not achieved for the intervention group $(\mathrm{N}=43)$. Therefore, the study might have been slightly underpowered. The sample size calculation was based on the assumption that postoperative morphine use would be reduced with one-third in the guided imagery group. Since postoperative mean morphine use was even a little higher in the guided imagery group compared to the control group (15.8 versus $12.5 \mathrm{mg}$ ), it is highly unlikely that seven more patients in the intervention group would have significantly altered the outcome of the study. Another limitation of the present study was the lack of documentation of other pain-related medication that patients may have used throughout the study period, such as paracetamol and diclofenac. Differences in consumption of these pain medications could have affected the outcome of surgery.

A systematic review on predictors of postoperative pain demonstrated that in all 15 included publications on this subject, a positive correlation was observed between preoperative anxiety and postoperative pain [4]. This was also the case in the control group of the present study, where a higher degree of anxiety was significantly correlated with a higher degree of pain. However, interestingly, within the guided imagery group no significant correlation could be observed between preoperative anxiety and postoperative pain. These findings indicate that guided imagery may have affected anxiety and pain levels to some extent in patients undergoing LC.

In summary, guided imagery did not significantly reduce preoperative anxiety and affect surgery outcome parameters such as morphine use, pain and patient satisfaction. It therefore seems not to be as simple as to provide patients with a CD before surgery in order to effectively self-manage postoperative pain. Since previous studies have demonstrated positive effects upon guided imagery, further research is needed before wide-spread implementation of this relaxation technique is advocated. Future studies may investigate which aspects of the guided imagery intervention, i.e. attention, information, suggestions, etc., affects surgical outcome parameters.

\section{Acknowledgement}

We thank all patients who participated in this study. The study was supported by the Slotervaart Hospital with respect to the development and provision of the guided imagery CD ("Gezonde Verbeelding"; Elsevier Publishers).

\section{References}

1. McGuire L, Heffner K, Glaser R, Needleman B, Malarkey W, et al. (2006) Pain and wound healing in surgical patients. Ann Behav Med 2: 165-172.

2. Morrison RS, Magaziner J, McLaughlin MA, Orosz G, Silberzweig SB, et al. (2003) The impact of post-operative pain on outcomes following hip fracture. Pain 3: 303-311.

3. Perkins FM and Kehlet $H(2000)$ Chronic pain as an outcome of surgery. A review of predictive factors. Anesthesiology 4: 1123-1133.

4. Ip HY, Abrishami A, Peng PW, Wong J, and Chung F (2009) Predictors of postoperative pain and analgesic consumption: A qualitative systematic review. Anesthesiology 3: 657-677.

5. Wasiak J, Cleland $\mathrm{H}$ (2007) Lidocaine for pain relief in burn injured patients Cochrane Database Syst Rev 3: Cd005622.

6. Saadat H, Drummond-Lewis J, Maranets I, Kaplan D, Saadat A, et al. (2006) Hypnosis reduces preoperative anxiety in adult patients. Anesth Analg 5: 1394 1396.

7. Berger MM, Davadant M, Marin C, Wasserfallen JB, Pinget C et al. (2010) Impact of a pain protocol including hypnosis in major burns. Burns 5: 639-646.

8. Morris LD, Louw QA, and Grimmer-Somers K (2009) The effectiveness of virtual reality on reducing pain and anxiety in burn injury patients: A systematic review. Clin J Pain 9: 815-826.

9. Holden-Lund C (1988) Effects of relaxation with guided imagery on surgical stress and wound healing. Res Nurs Health 4: 235-244.

10. Lambert SA (1996) The effects of hypnosis/guided imagery on the postoperative course of children. J Dev Behav Pediatr 5: 307-310.

11. Heinschel JA (2002) A descriptive study of the interactive guided imagery experience. J Holist Nurs 4: 325-346.

12. Schulz KF, Altman DG, Moher D (2010) CONSORT 2010 Statement: Updated guidelines for reporting parallel group randomised trials. Trials 11: 32 .

13. Moerman N, van Dam FS, Muller MJ, Oosting H (1996) The Amsterdam preoperative anxiety and information scale (APAIS). Anesth Analg 3: 445-451. 
Citation: Pijl AJ, de Gast HM, Jong M, Hoen MB, Kluyver EB, et al. (2016) Guided Imagery Intervention does not Affect Surgical Outcome of Patients Undergoing laparoscopic Cholecystectomy: A Multi-Centre, Randomised Controlled Study. J Pat Care 2: 119. doi: 10.4172/25734598.1000119

Page 5 of 5

14. Marshall GN, Hays RD, Sherbourne CB, Wells KB (1993) The structure of patient satisfaction with outpatient medical care. Psychological Assessment 5 : 477-483.

15. Broadbent E, Kahokehr A, Booth RJ, Thomas J, Windsor JA, et al. (2012)
A brief relaxation intervention reduces stress and improves surgical wound healing response: A randomised trial. Brain Behav Immun 2: 212-217.

16. Fernandez MD, Luciano C, Valdivia-Salas S (2012) Impact of acceptancebased nursing intervention on postsurgical recovery: Preliminary findings. Span J Psychol 3: 1361-1370. 